\title{
High intensity intermittent exercise improves cardiac structure and function and reduces liver fat in patients with type 2 diabetes: a randomised controlled trial
}

\author{
Sophie Cassidy ${ }^{1,3} \cdot$ Christian Thoma $^{1} \cdot$ Kate Hallsworth $^{1,3}$ - Jehill Parikh ${ }^{1,2}$. \\ Kieren G. Hollingsworth ${ }^{1,2}$ • Roy Taylor ${ }^{1,2}$ • Djordje G. Jakovljevic ${ }^{1,3}$ • \\ Michael I. Trenell ${ }^{1,2,3}$
}

Received: 20 May 2015 / Accepted: 5 August 2015 /Published online: 9 September 2015

(C) The Author(s) 2015. This article is published with open access at Springerlink.com

\begin{abstract}
Aims/hypothesis Cardiac disease remains the leading cause of mortality in type 2 diabetes, yet few strategies to target cardiac dysfunction have been developed. This randomised controlled trial aimed to investigate high intensity intermittent training (HIIT) as a potential therapy to improve cardiac structure and function in type 2 diabetes. The impact of HIIT on liver fat and metabolic control was also investigated.

Methods Using an online random allocation sequence, 28 patients with type 2 diabetes (metformin and diet controlled) were randomised to 12 weeks of HIIT $(n=14)$ or standard care $(n=14)$. Cardiac structure and function were measured by $3.0 \mathrm{~T}$ MRI and tagging. Liver fat was determined by ${ }^{1} \mathrm{H}$-magnetic resonance spectroscopy and glucose control by an OGTT. MRI analysis was performed by an observer blinded to group allocation. All study procedures took place in Newcastle upon Tyne, UK.

Results Five patients did not complete the study and were therefore excluded from analysis: this left 12 HIIT and 11 control patients for the intention-to-treat analysis. Compared with controls, HIIT improved cardiac structure (left ventricular wall mass $104 \pm 17 \mathrm{~g}$ to $116 \pm 20 \mathrm{~g}$ vs $107 \pm 25 \mathrm{~g}$ to $105 \pm 25 \mathrm{~g}$,
\end{abstract}

Michael I. Trenell

michael.trenell@ncl.ac.uk

Institute of Cellular Medicine, Newcastle University, Newcastle upon Tyne NE2 4HH, UK

2 Magnetic Resonance Centre, Campus for Ageing and Vitality, Newcastle University, Newcastle upon Tyne NE4 5PL, UK

3 UKRC Centre for Ageing and Vitality, Faculty of Medical Sciences, Newcastle University, Newcastle upon Tyne NE2 4HH, UK $p<0.05$ ) and systolic function (stroke volume $76 \pm 16 \mathrm{ml}$ to $87 \pm 19 \mathrm{ml}$ vs $79 \pm 14 \mathrm{ml}$ to $75 \pm 15 \mathrm{ml}, p<0.01)$. Early diastolic filling rates increased $(241 \pm 84 \mathrm{ml} / \mathrm{s}$ to $299 \pm 89 \mathrm{ml} / \mathrm{s}$ vs $250 \pm 44 \mathrm{ml} / \mathrm{s}$ to $251 \pm 47 \mathrm{ml} / \mathrm{s}, p<0.05)$ and peak torsion decreased $\left(8.1 \pm 1.8^{\circ}\right.$ to $6.9 \pm 1.6^{\circ}$ vs $7.1 \pm 2.2^{\circ}$ to $7.6 \pm 1.9^{\circ}$, $p<0.05)$ in the treatment group. Following HIIT, there was a $39 \%$ relative reduction in liver fat $(p<0.05)$ and a reduction in $\mathrm{HbA}_{1 \mathrm{c}}(7.1 \pm 1.0 \%$ [54.5 $\mathrm{mmol} / \mathrm{mol}]$ to $6.8 \pm 0.9 \%$ [51.3 $\mathrm{mmol} / \mathrm{mol}]$ vs $7.2 \pm 0.5 \%$ [54.9 $\mathrm{mmol} / \mathrm{mol}]$ to $7.4 \pm 0.7 \%$ [57.0 mmol $/ \mathrm{mol}], p<0.05)$. Changes in liver fat correlated with changes in $\mathrm{HbA}_{1 \mathrm{c}}(r=0.70, p<0.000)$ and $2 \mathrm{~h}$ glucose $(r=$ $0.57, p<0.004)$. No adverse events were recorded.

Conclusions/interpretation This is the first study to demonstrate improvements in cardiac structure and function, along with the greatest reduction in liver fat, to be recorded following an exercise intervention in type 2 diabetes. HIIT should be considered by clinical care teams as a therapy to improve cardiometabolic risk in patients with type 2 diabetes.

Trial registration: www.isrctn.com 78698481

Funding: Medical Research Council.

Keywords Fatty liver $\cdot$ Heart diseases $\cdot$ Left ventricle $\cdot$ MRI . Type 2 diabetes mellitus

$\begin{array}{ll}\text { Abbreviations } \\ \text { ALP } & \text { Alanine phosphatase } \\ \text { ALT } & \text { Alanine transaminase } \\ \text { AST } & \text { Aspartate aminotransferase } \\ \text { AUGC } & \text { Area under the glucose curve } \\ \text { HIIT } & \text { High intensity intermittent training } \\ \text { PCr } & \text { Phosphocreatine }\end{array}$


RPE Rating of perceived exertion

$\dot{V} \mathrm{O}_{2 \text { peak }}$ Peak oxygen consumption

\section{Introduction}

Heart disease is the leading cause of morbidity and mortality in type 2 diabetes [1], and more than a quarter of all hospital admissions for heart failure in the West involve a patient with diabetes [2, 3]. Early changes in left ventricular structure and function have been identified in adults with type 2 diabetes prior to any overt cardiac disease. These include pathological hypertrophy [4], reduced end-diastolic blood volume [5], diastolic and systolic dysfunction [5-7], and alterations in strain patterns $[6,8]$, as identified using sensitive magnetic resonance techniques. MRI measurement has been shown to have greater reproducibility than two-dimensional echocardiography in healthy and failing hearts, while avoiding the ionising radiation exposure associated with computed tomography methods, permitting ethical longitudinal studies [9]. Despite clear cardiac dysfunction in type 2 diabetes, therapies to target these preclinical cardiac changes are sparse.

Treatment algorithms for type 2 diabetes support a physically active lifestyle at every stage of treatment [10]. Indeed, aerobic and resistance exercises have known benefits to cardiovascular function [11], although little is known about the impacts on cardiac structure and function. More recently, attention has been given to the intensity of exercise, with high intensity intermittent training (HIIT) fast becoming a popular alternative to continuous moderate exercise [12]. HIIT refers to brief intervals of vigorous activity interspersed with periods of low activity or rest, and elicits a strong cardiac response compared with moderate continuous exercise $[12,13]$. It therefore has the potential to improve cardiac structure and function in type 2 diabetes. However, the cardiac benefits of HIIT in type 2 diabetes are still to be determined, and the metabolic effects are also unclear. Only two 12 week HIIT studies have shown improvements in $\mathrm{HbA}_{1 \mathrm{c}}[14,15]$, and the impact of HIIT on ectopic fat (which plays an important role in glucose homeostasis $[16,17]$ ) has not been investigated. We therefore aimed to define the effect of HIIT on cardiac structure and function, regional fat deposition, and glycaemic control in people with type 2 diabetes. We hypothesised that HIIT would improve cardiac structure and function, alongside improving glycaemic control and reducing ectopic fat, in adults with type 2 diabetes.

\section{Methods}

Patients Type 2 diabetic patients (stable control with diet and/or metformin for at least 6 months) were randomised to a HIIT $(n=14)$ or a control group $(n=14)$. Participant characteristics can be seen in Table 1. Participants were excluded if they had any overt cardiac disease, took part in regular exercise $(\geq 60 \mathrm{~min}$ moderate-vigorous activity per week), were being treated with $\beta$-blocker medication or had any contraindications to exercise stress testing according to guidelines [18]. The study was approved by the Newcastle and Northeast Tyneside Local Research Ethics Committee and all participants provided written informed consent. Participants were recruited via advertising in local newspapers and diabetes community groups between September 2012 and September 2013. During the study, two participants left for unrelated medical reasons, one participant could not commit the time and two failed to comply with MRI procedures, leaving 12 in the HIIT group and 11 in the control group (Fig. 1).

Experimental protocol and randomisation Following an initial screening visit, cardiac structure and function, liver and visceral fat, body composition, glycaemic control and blood variables were measured at baseline and after 12 weeks of HIIT or continued standard care. Glucose control was measured within $48-72 \mathrm{~h}$ of the final exercise session to control for the acute effect of exercise on glucose uptake. Patients were randomised into groups using a simple random allocation sequence (www.randomization.com). Concealed envelopes with consecutive numbers were locked in a drawer and withdrawn in numerical order by the main author (SC).

Screening To determine underlying cardiac disease, a medical history, physical examination, and resting and exercise 12-lead

Table 1 Participant characteristics

\begin{tabular}{|c|c|c|c|}
\hline Characteristic & Control group & HIIT group & $p$ value \\
\hline$n$ (men/women) & $8 / 3$ & $10 / 2$ & \\
\hline Age (years) & $59 \pm 9$ & $61 \pm 9$ & 0.70 \\
\hline Time since diagnosis (years) & $4 \pm 2$ & $5 \pm 3$ & \\
\hline BMI $\left(\mathrm{kg} / \mathrm{m}^{2}\right)$ & $32 \pm 6$ & $31 \pm 5$ & 0.71 \\
\hline Height (cm) & $169 \pm 9$ & $171 \pm 8$ & 0.71 \\
\hline Weight (kg) & $90 \pm 9$ & $90 \pm 15$ & 0.95 \\
\hline $\mathrm{HbA}_{1 \mathrm{c}}(\%)$ & $7 \pm 0.5$ & $7 \pm 1$ & 0.87 \\
\hline $\mathrm{HbA}_{1 \mathrm{c}}(\mathrm{mmol} / \mathrm{mol})$ & $55 \pm 6$ & $54 \pm 11$ & 0.88 \\
\hline Fasting glucose (mmol/l) & $7.0 \pm 1.0$ & $6.8 \pm 1.6$ & 0.69 \\
\hline $2 \mathrm{~h}$ glucose $(\mathrm{mmol} / \mathrm{l})$ & $11.7 \pm 3.1$ & $12.5 \pm 3.1$ & 0.57 \\
\hline Liver fat $(\%)$ & $7.1 \pm 6.8$ & $6.9 \pm 6.9$ & 0.94 \\
\hline$\dot{V} \mathrm{O}_{2 \text { peak }}\left(\mathrm{ml} \mathrm{kg}^{-1} \min ^{-1}\right)$ & $20.3 \pm 6.1$ & $21.8 \pm 5.4$ & 0.54 \\
\hline \multicolumn{4}{|l|}{ Medications } \\
\hline Metformin & 7 & 7 & \\
\hline Statins & 6 & 7 & \\
\hline BP & 5 & 3 & \\
\hline
\end{tabular}

$\dot{\mathrm{V}} \mathrm{O}_{2 \text { peak }}$ is normalised to body mass 
Fig. 1 Consort flow diagram showing patient numbers at each stage of the trial

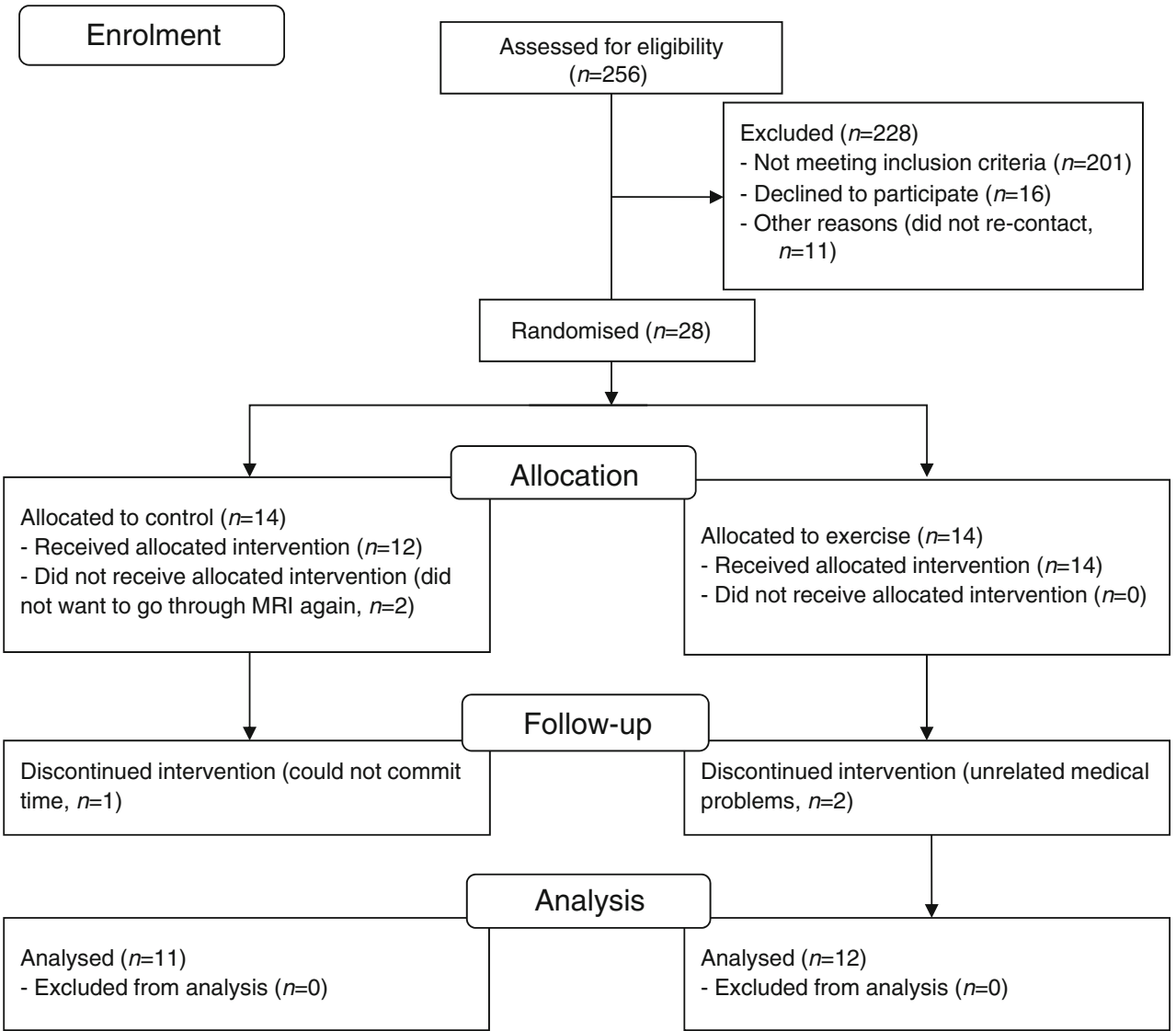

electrocardiography (Custo med, Ottobrunn, Germany) were performed. During the exercise test, gas exchange was measured to determine peak oxygen consumption $\left(\dot{V} \mathrm{O}_{2 \text { peak }}\right)$ normalised to body mass. $\dot{V} \mathrm{O}_{2 \text { peak }}$ was determined as the point at which participants reached volitional exhaustion, participants could no longer maintain a cycling rate of $60 \mathrm{rev} / \mathrm{min}$, or continuing exercise was contraindicated [18]. The test was performed using an electronically braked semirecumbent cycle ergometer (Corival Lode, Groningen, The Netherlands) and resistance was increased by $1 \mathrm{~W}$ every $6 \mathrm{~s}$. Before the exercise test, patients completed a 20 min resting period in which they lay supine while beat-to-beat BP was measured by the vascular unloading technique [19].

Cardiac MRI All examinations were performed using the 3.0 T Philips Achieva MRI scanner with a six channel cardiac array (Philips Medical Systems, Best, The Netherlands). During breath holding, a stack of balanced steady-state free precession images were obtained in the short axis view, covering the entire left ventricle (field of view $[\mathrm{FOV}]=350 \mathrm{~mm}$, repetition time $[\mathrm{TR}] /$ echo time $[\mathrm{TE}]=3.7 / 1.9 \mathrm{~ms}$, turbo factor 17 , flip angle $40^{\circ}$, slice thickness $8 \mathrm{~mm}, 0 \mathrm{~mm}$ gap, 14 slices, 25 phases, resolution $1.84 \times 1.37 \mathrm{~mm}$ with zero filling to $1.37 \times 1.37 \mathrm{~mm}$ and temporal duration approximately $40 \mathrm{~ms}$ per phase, dependent on heart rate). Using a Viewforum workstation (Philips Medical Systems), the short axis slices at end-diastole and end-systole were used (Fig. 2a) to manually trace endocardial and epicardial borders, with papillary muscles excluded from volume calculations but included in calculations of left ventricular mass. The apical slice was defined as the last slice showing inter-cavity blood pools, and the basal slice as the last slice in which at least $50 \%$ of the blood volume was surrounded by myocardium. The analysis was performed by a single observer blinded to group allocation. Details of the algorithm for contour selection and calculation of left ventricular mass, systolic and diastolic variables have been previously published [20]. The eccentricity ratio is a measure of concentric remodelling, and was calculated by dividing left ventricular mass by end-diastolic blood volume. Longitudinal shortening was determined from cine MRI in the four-chamber view by determining the perpendicular distance from the plane of the mitral valve to the apex in systole and diastole.

Cardiac tagging Tagged short axis images were acquired. Cardiac tagging works by applying radiofrequency pulses to cancel the MR signal from the myocardium in diastole in a rectangular grid pattern and tracking the deformation of these tags through the rest of the cardiac cycle (Fig. 2a). A turbo-field echo sequence with acceleration factor 9 was used $(\mathrm{TR}=4.9$, 
a

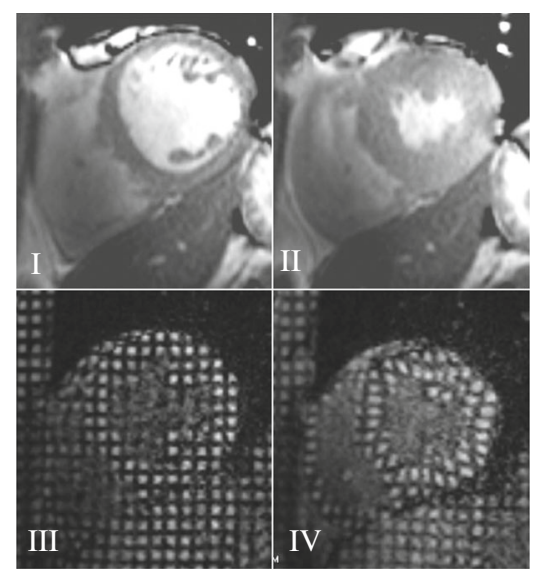

b

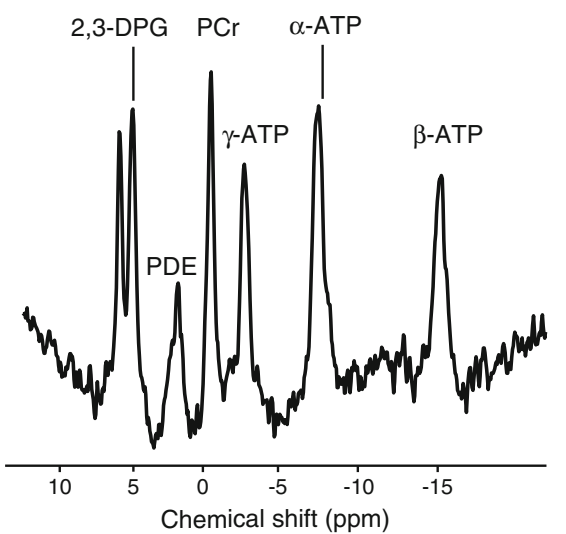

Fig. 2 Cardiac MRI techniques. These include (a) Cardiac cine imaging (I, II) and cardiac tagging (III, IV) at diastole (I, III) and systole (II, IV), showing how a rectangular grid of nulled signal applied at diastole remains with the tissue through the cardiac cycle, allowing calculation of strain and torsion. (b) Phosphorus spectroscopy. Spectrum presented before correction for saturation due to blood content, flip angle at the cardiac tissue and heart rate. DPG, diphosphoglycerate; PDE, phosphodiesters

$\mathrm{TE}=3.1$, flip angle $=10^{\circ}$, number of excitations $=1$, SENSE factor 2 , FOV $350 \times 350 \mathrm{~mm}$, voxel size $1.37 \times 1.37 \mathrm{~mm}$ with no zero filling and an orthogonal complementary spatial modulation of magnetistion grid with tag spacing of $7 \mathrm{~mm}$ ). Short axis slices of $10 \mathrm{~mm}$ thickness were prescribed. The Cardiac Image Modelling package (University of Auckland, New Zealand) was used to analyse the tagging data by aligning a mesh on the tags between the endo- and epicardial contours. Details of the calculation of strain and torsion variables have been previously published [21].

Cardiac spectroscopy Cardiac high-energy phosphate metabolism was assessed using ${ }^{31} \mathrm{P}$-magnetic resonance spectroscopy (see Fig. $2 \mathrm{~b}$ for an example cardiac spectrum). Cardiac acquisition was vectorcardiogram-gated with the participant in a prone position during free breathing, with a $10 \mathrm{~cm}$ diameter ${ }^{31} \mathrm{P}$ surface coil used for transmission/reception (PulseTeq,
Chobham, UK) and the scanner body coil used for localising images. A cardiac gated one-dimensional chemical shift imaging sequence was used, and to eliminate contamination from liver, a $7 \mathrm{~cm}$ slice was selected in the foot-head direction using a selective pulse (of the 'spredrex' type, as outlined in [22]). The flip angle subtended at the myocardium was fixed to achieve $50^{\circ}$ excitation at the target depth and measured using a variable flip angle sequence on a gadolinium-doped $20 \mathrm{mmol} / \mathrm{l}$ phenyl phosphonic acid phantom at the centre of the coil. Sixteen coronal phase-encoding steps yielded spectra from $10 \mathrm{~mm}$ slices $(\mathrm{TR}=$ heart rate, 192 averages, acquisition time approximately $20 \mathrm{~min}$ ), using a trigger delay of $400 \mathrm{~ms}$. A cosine apodisation filter was applied. The first spectrum containing signal beyond the chest wall and solely from cardiac tissue was selected. The spectrum was analysed using an AMARES time domain fit algorithm in jMRUI [23] and the ATP peak area was corrected for blood contamination [24].

Liver and visceral fat MRI Liver fat was assessed by ${ }^{1} \mathrm{H}$-magnetic resonance spectroscopy $(\mathrm{TR} / \mathrm{TR}=3,000 \mathrm{~ms} / 35$, $50,75,100,125$ or $150 \mathrm{~ms}, 3 \times 3 \times 3 \times 3 \mathrm{~cm}$ voxel, SENSE torso array, one signal average and an expiration breath hold of $17 \mathrm{~s}$ ). The water and $\mathrm{CH}_{2}$ resonances were analysed using the AMARES algorithm in jMRUI [23]. The T2 relaxation times of the water and $\mathrm{CH}_{2}$ resonances for each participant were calculated from the data by monoexponential fitting of signal intensity vs the six echo times, the signals at $35 \mathrm{~ms}$ were then corrected for $\mathrm{T} 2$ decay and the fat fraction was calculated as a percentage of the total signal from that volume [25].

Visceral fat was estimated at the L4-L5 junction [26] using a three-point Dixon sequence $(\mathrm{TR}=50 \mathrm{~ms}, \mathrm{TE}=3.45$, 4.60 or $5.75 \mathrm{~ms}$, number of averages $=1$, flip angle $=5^{\circ}$, voxel size $2.5 \times 2.5 \mathrm{~mm}$, slice thickness $10 \mathrm{~mm}$, zero filled to $1.4 \times 1.4 \mathrm{~mm}$, and median FOV $440 \mathrm{~mm}$ [range $400-480 \mathrm{~mm}$ to suit participant size], with $70 \%$ phase FOV) [27]. Binary gating and a watershed algorithm was used to divide the binary image into distinct areas (Fig. 3); this allowed easy separation and quantification of the subcutaneous and visceral fat areas using ImageJ [28]. Liver and visceral fat analyses were performed by a single observer blinded to group allocation.

Glycaemic control, blood variables and body composition After an $8 \mathrm{~h}$ minimum overnight fast, a $75 \mathrm{~g}$ OGTT was performed in which samples were drawn every $15 \mathrm{~min}$ and analysed for whole blood glucose (YSI 2300 Stat Plus-D, Yellow Springs Instruments, Yellow Springs, OH, USA) and plasma insulin (Mercodia Iso-Insulin ELISA, no. 10-1128-01, Mercodia, Uppsala, Sweden). Insulin resistance and beta cell function were predicted using the HOMA2 [29], and the area under the glucose curve (AUGC) was calculated using the trapezoidal rule [30]. Fasting plasma samples were analysed in an accredited clinical pathology laboratory (Department of Clinical Biochemistry, Newcastle upon Tyne Hospital NHS 

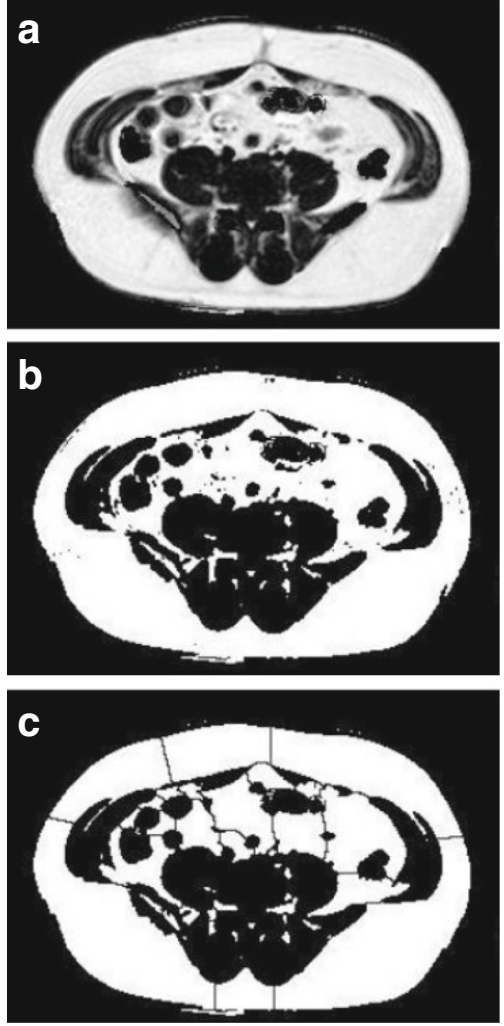

Fig. 3 Quantifying visceral fat. (a) The three-point Dixon fat fraction map acquired at the L4-L5 junction. (b) Binary thresholding of structures containing more than $50 \%$ fat (visceral and subcutaneous fat) from those with less. Total area is evaluated. (c) Application of thresholding algorithm divides segmented image into chunks and separates visceral and subcutaneous fat around the boundary of the chest wall. Selection of subcutaneous fat and any external signals allows measurement of this area and subtraction from the total to yield visceral fat area

Foundation Trust) for alanine phosphatase (ALP), alanine transaminase (ALT), aspartate aminotransferase (AST), total cholesterol, triacylglycerols and $\mathrm{HbA}_{1 \mathrm{c}}$. Total cholesterol, triacylglycerols, ALP, AST and ALT were measured using a Roche P800 Modular Analyzer (Basel, Switzerland) and $\mathrm{HbA}_{1 \mathrm{c}}$ was measured using a TOSOH HLC-723G8 (Minato, Tokyo, Japan). Body composition was measured using air displacement plethysmography (BodPod, Life Measurement, CA, USA).

Intervention The HIIT group performed 36-cycle ergometry sessions over 12 weeks (3 sessions per week on nonconsecutive days) at a local gym. Patients were required to perform at least 32 sessions ( $89 \%$ of total sessions) for inclusion in the analysis. Intensity was based on the 6-20 point Borg Rating of Perceived Exertion (RPE) [31]. Each session included a $5 \mathrm{~min}$ warm up in which participants would progress from RPE 9 to 13 ('very light' to 'somewhat hard'), followed by five intervals, each with a pedal cadence of $>80$ rev/min, reaching a RPE 16-17 ('very hard'). The final interval was then followed by a 3 min recovery cycle. Intervals lasted 2 min in week 1 and progressed by $10 \mathrm{~s}$ increments each week such that week 12 consisted of 3 min $50 \mathrm{~s}$ intervals. Three min recovery periods interspersed each interval, which consisted of $90 \mathrm{~s}$ passive recovery, $60 \mathrm{~s}$ of band-resisted upper body exercise and $30 \mathrm{~s}$ to prepare for the subsequent interval. The arm resistance bands (Bodymax Fitness, Clydebank, UK) were used as light recovery and involved one exercise per recovery period in the following order: face pull, horizontal push, horizontal pull and $30^{\circ}$ push. The initial session was supervised; thereafter, participants were guided through each session by voice-recorded instructions using an iPod shuffle (Apple, CA, USA). An exercise diary was completed to monitor exercise adherence.

Apart from HIIT sessions, all study participants were instructed to continue their normal routine and care for 12 weeks and not to change medication, habitual physical activity, diet or body weight. Weekly phone calls were made to assess adherence, and habitual physical activity was assessed over 7 days pre- and post-intervention using a validated multisensory armband (Sensewear; Bodymedia, Pittsburgh, PA, USA) [32].

Statistical analysis Statistical power was based on the change in $\mathrm{HbA}_{1 \mathrm{c}}$. We selected a sample size of 12 to provide a statistical power of $80 \%$ to detect a difference of $0.6 \%$ in $\mathrm{HbA}_{1 \mathrm{c}}$ [33]. A sample size of 14 was used to allow for two dropouts per group. A per-protocol analysis was adopted because the intention of this study was to assess efficacy and mechanisms of change, not effectiveness. All analyses were performed using IBM SPSS Statistics software (version 19, NY, USA) and data are presented as means \pm SD unless otherwise stated. Continuous data were tested for normality using the Shapiro-Wilk test. Comparisons of key baseline variables were made using independent sample $t$ tests. Between-group comparisons were made using ANCOVA with the baseline value as the covariate. Within-group changes were assessed by paired-sample $t$ tests or the non-parametric alternative (Wilcoxon signed-rank test) for non-normally distributed data. Adjustment for multiple comparisons was not made because of co-linearity between variables, hypothesis-driven comparisons and the increased risk of type II error following adjustment [34]. Pearson's correlation or the non-parametric alternative (Spearman's rank correlation) was used to calculate $r$ values among body composition, metabolic and cardiac variables. $P$ values $<0.05$ were considered statistically significant.

\section{Results}

The groups were matched well for all baseline characteristics (Table 1). Glycaemic control was similar between groups and liver fat was above the clinically defined threshold for nonalcoholic fatty liver disease $(>5 \%)$ in both groups. Adherence 
to intervention was good, with HIIT patients completing an average of $36 \pm 0.9$ sessions, and Sensewear armband activity revealed no within-group change in habitual physical activity (daily energy expenditure: HIIT $2,701 \pm 299$ to $2,537 \pm 386$, $p=0.129$ vs control $2,548 \pm 366$ to $2,455 \pm 166, p=0.459$ (calories)).

Cardiac structure, function and energetics HIIT induced structural cardiac changes, with a $12 \%$ relative increase in left ventricular wall mass $(p<0.05)$ and increased end-diastolic blood volume $(p<0.01$; Fig. $4 \mathrm{a})$. The exercise group also demonstrated improvements in systolic function, indicated by raised stroke volume $(p<0.01)$ and left ventricular ejection fraction $(p<0.05)$. The early diastolic filling rate increased by
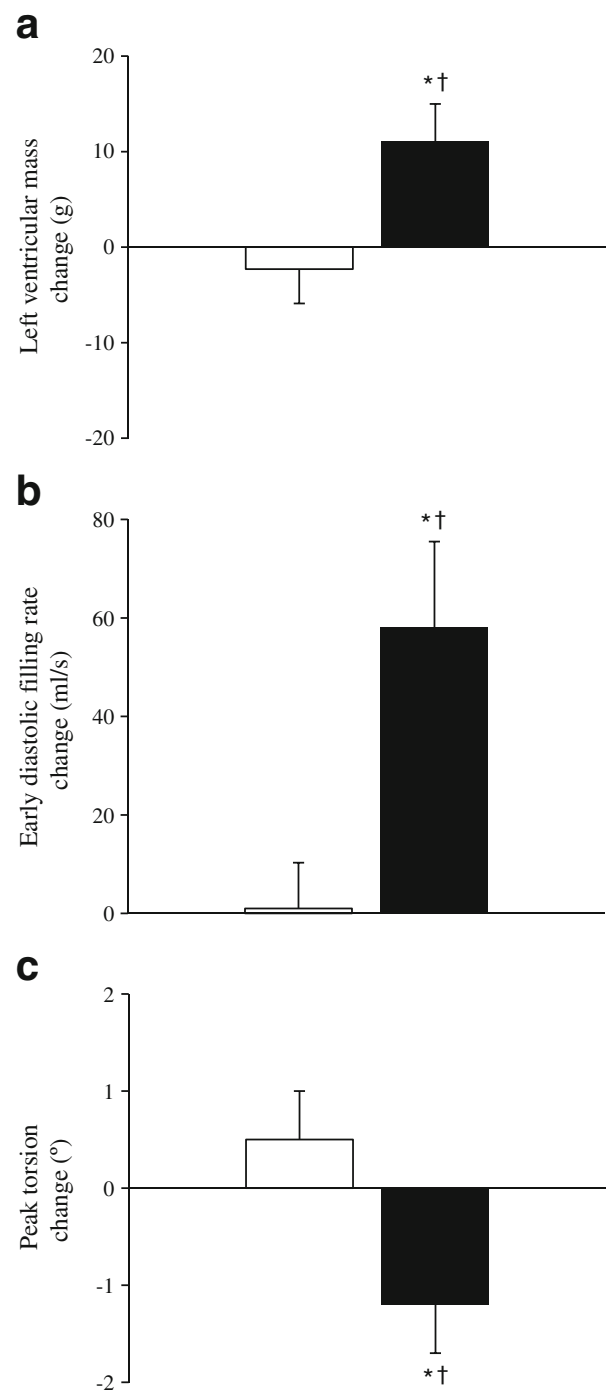

Fig. 4 Effect of HIIT vs control on (a) left ventricular mass, (b) diastolic filling rate and (c) peak torsion. White bars, control; black bars, HIIT. Values are means \pm SEM. *Significant difference baseline vs post-treatment $(p<0.05)$. ${ }^{\dagger}$ Significant difference between-group interaction $(p<0.05)$
$24 \%$ (Fig. 4b), and within-group comparison revealed a significant increase in early filling percentage after HIIT $(57 \pm 9 \%$ to $60 \pm 9 \%, p<0.05$; Table 2 ). There was a $15 \%$ relative decrease in peak torsion after exercise $\left(8.1 \pm 1.8^{\circ}\right.$ to $6.9 \pm 1.6^{\circ}$ vs $7.1 \pm 2.2^{\circ}$ to $7.6 \pm 1.9^{\circ} ; p<0.05$; Fig. $4 \mathrm{c}$ ); myocardial strain remained constant. The phosphocreatine $(\mathrm{PCr}) / \mathrm{ATP}$ ratio did not change following HIIT ( $p=0.115$; Table 2$)$.

Body composition Within-group comparisons revealed no change in body weight after exercise. However, the $1 \%$ increase and decrease in control and HIIT, respectively, was a significant between-group interaction $(p<0.05$; Table 3$)$. There was no effect of HIIT on whole body fat mass but within-group comparison revealed a reduction in visceral adipose tissue $\left(201 \pm 80 \mathrm{~cm}^{2}\right.$ to $181 \pm 72 \mathrm{~cm}^{2}, p<0.05$; Table 3). The change in whole body fat mass (in $\mathrm{kg}$ ) was associated with changes in $2 \mathrm{~h}$ glucose $(r=0.46, p=0.027)$ and $\mathrm{HbA}_{1 \mathrm{c}}(r=0.60, p=0.003)$.

Liver fat and enzymes HIIT elicited a 39\% relative reduction in liver fat $(6.9 \pm 6.9 \%$ to $4.2 \pm 3.6 \%, p<0.05)$ so that four patients in the exercise group moved from having clinically significant liver fat to within 'normal' limits $(<5 \%$, Table 3$)$. There was a significant between-group interaction for HIIT and liver fat ( $p<0.05$; Table 3$)$, accompanied by withingroup changes in ALT and AST $(p<0.5$; Table 3$)$, both markers of liver damage. Change in liver fat correlated with change in fasting glucose $(r=0.45, p=0.030), 2 \mathrm{~h}$ glucose $(r=0.57, p=0.004)$ and $\mathrm{HbA}_{1 \mathrm{c}}(r=0.70, p=0.000)$.

Glycaemic control HIIT had no impact on fasting glucose $(6.8 \pm 1.6 \mathrm{mmol} / 1$ to $6.8 \pm 1.6 \mathrm{mmol} / 1, p=0.866)$ or fasting insulin (65.5 $\pm 39.5 \mathrm{pmol} / 1$ to $65.5 \pm 32.8 \mathrm{pmol} / 1, p=0.875)$; however, between-group comparisons revealed improvements in $\mathrm{HbA}_{1 \mathrm{c}}, 2 \mathrm{~h}$ glucose and AUGC ( $p<0.05$; Table 3$)$. There was no improvement in insulin sensitivity (HOMA2 of insulin resistance [HOMA2-IR] and HOMA2 of insulin sensitivity [HOMA2-\%S]) or beta cell function (HOMA2 of beta cell function [HOMA2- $\beta]$ ).

\section{Discussion}

This is the first study to examine the effects of HIIT on cardiac structure and function, regional fat deposition, and glycaemic control in people with type 2 diabetes. The main findings were that a 12 week HIIT programme increased left ventricular wall mass and end-diastolic blood volume, improved systolic and diastolic function, reduced peak torsion, and decreased liver fat. Therefore, HIIT was an effective strategy to reverse cardiac dysfunction and reduce liver fat in this patient group and was accompanied by modest improvements in glycaemic control. 
Table 2 Effect of HIIT on cardiac structure, function and metabolism

\begin{tabular}{|c|c|c|c|c|c|c|c|}
\hline \multirow[t]{2}{*}{ Parameter } & \multicolumn{3}{|l|}{ Control } & \multicolumn{3}{|l|}{ HIIT } & \multirow{2}{*}{$\begin{array}{l}\text { Adjusted between- } \\
\text { group } p \text { value }\end{array}$} \\
\hline & Pre & Post & $\begin{array}{l}\text { Within- } \\
\text { group } p \\
\text { value }^{\mathrm{a}}\end{array}$ & Pre & Post & $\begin{array}{l}\text { Within- } \\
\text { group } p \\
\text { value }^{\mathrm{a}}\end{array}$ & \\
\hline \multicolumn{8}{|l|}{ Cardiac structure } \\
\hline Left ventricular wall mass (g) & $107 \pm 25$ & $105 \pm 25$ & 0.54 & $104 \pm 17$ & $116 \pm 20$ & $0.02^{*}$ & $0.03^{\dagger}$ \\
\hline Wall thickness systole (mm) & $5.5 \pm 1.1$ & $6.2 \pm 1.0$ & $0.01 * *$ & $6.2 \pm 1.5$ & $6.8 \pm 1.1$ & 0.07 & 0.54 \\
\hline Wall thickness diastole (mm) & $9.1 \pm 2.5$ & $10.1 \pm 2.5$ & $0.02 *$ & $10.7 \pm 3.1$ & $11.5 \pm 1.8$ & 0.32 & 0.43 \\
\hline Eccentricity ratio $(\mathrm{g} / \mathrm{ml})$ & $0.85 \pm 0.24$ & $0.87 \pm 0.18$ & 0.66 & $0.94 \pm 0.28$ & $0.96 \pm 0.24$ & 0.70 & 0.66 \\
\hline End-diastolic volume (ml) & $129 \pm 28$ & $122 \pm 28$ & 0.08 & $118 \pm 30$ & $126 \pm 30$ & $0.01 * *$ & $0.00^{\dagger \dagger}$ \\
\hline End-systolic volume (ml) & $50 \pm 22$ & $47 \pm 22$ & 0.33 & $42 \pm 17$ & $39 \pm 13$ & 0.25 & 0.76 \\
\hline \multicolumn{8}{|l|}{ Systolic function } \\
\hline Systolic BP (mmHg) & $126 \pm 3$ & $124 \pm 5$ & 0.62 & $123 \pm 4$ & $122 \pm 4$ & 0.66 & 0.99 \\
\hline Diastolic BP (mmHg) & $84 \pm 2$ & $80 \pm 2$ & 0.07 & $81 \pm 2$ & $80 \pm 2$ & 0.81 & 0.41 \\
\hline Heart rate (bpm) & $63 \pm 7$ & $69 \pm 13$ & 0.21 & $67 \pm 12$ & $66 \pm 16$ & 0.69 & 0.27 \\
\hline Stroke volume (ml) & $79 \pm 14$ & $75 \pm 15$ & 0.16 & $76 \pm 16$ & $87 \pm 19$ & $0.00 * *$ & $0.00^{\dagger \dagger}$ \\
\hline Cardiac output (1/min) & $5.0 \pm 1.0$ & $5.2 \pm 1.0$ & 0.54 & $5.0 \pm 1.00$ & $5.5 \pm 1.0$ & 0.07 & 0.31 \\
\hline Ejection fraction (\%) & $64 \pm 11$ & $63 \pm 10$ & 0.62 & $65 \pm 8$ & $70 \pm 6$ & $0.02 *$ & $0.03^{\dagger}$ \\
\hline Longitudinal shortening (\%) & $13.1 \pm 2.2$ & $12.7 \pm 2.6$ & 0.62 & $12.2 \pm 3.0$ & $13.4 \pm 1.8$ & 0.28 & 0.39 \\
\hline \multicolumn{8}{|l|}{ Diastolic function } \\
\hline Early filling percentage (\%) & $58 \pm 11$ & $59 \pm 8$ & 0.88 & $57 \pm 9$ & $60 \pm 9$ & $0.04 *$ & 0.45 \\
\hline Early diastolic filling rate $(\mathrm{ml} / \mathrm{s})$ & $250 \pm 44$ & $251 \pm 47$ & 0.68 & $241 \pm 84$ & $299 \pm 89$ & $0.01 * *$ & $0.02^{\dagger}$ \\
\hline Late diastolic filling rate $(\mathrm{ml} / \mathrm{s})$ & $310 \pm 143$ & $285 \pm 60$ & 0.68 & $278 \pm 67$ & $289 \pm 64$ & 0.53 & 0.56 \\
\hline \multicolumn{8}{|l|}{ Strain and torsion } \\
\hline Peak endocardial circumferential strain (\%) & $23.1 \pm 4.1$ & $23.4 \pm 4.3$ & 0.82 & $25.2 \pm 4.6$ & $24.5 \pm 5.1$ & 0.61 & 0.82 \\
\hline Peak whole wall circumferential strain (\%) & $16.5 \pm 3.1$ & $16.0 \pm 3.3$ & 0.46 & $16.5 \pm 3.1$ & $16.4 \pm 4.0$ & 0.94 & 0.73 \\
\hline Peak torsion $\left({ }^{\circ}\right)$ & $7.1 \pm 2.2$ & $7.6 \pm 1.9$ & 0.19 & $8.1 \pm 1.8$ & $6.9 \pm 1.6$ & $0.04 *$ & $0.04^{\dagger}$ \\
\hline \multicolumn{8}{|l|}{ Metabolism } \\
\hline $\mathrm{PCr} / \mathrm{ATP}$ ratio & $1.76 \pm 0.51$ & $1.72 \pm 0.36$ & 0.80 & $1.74 \pm 0.39$ & $2.00 \pm 0.36$ & 0.19 & 0.12 \\
\hline
\end{tabular}

Data are mean \pm SD

${ }^{\text {a }}$ Paired $t$ test

${ }^{\mathrm{b}}$ Adjusted for baseline value for ANCOVA

*Significant difference baseline vs post-treatment $(p<0.05)$

**Significant difference baseline vs post-treatment $(p<0.01)$

${ }^{\dagger}$ Significant difference between-group interaction $(p<0.05)$

${ }^{\dagger}$ Significant difference between-group interaction $(p<0.01)$

bpm, beats per minute; pre, pre-treatment; post, post-treatment

Cardiac changes Left ventricular wall mass and enddiastolic blood volume increased after 12 weeks of HIIT. This 'physiological hypertrophy' is a known effect of exercise but should not be confused with 'pathological hypertrophy', as seen in those with type 2 diabetes $[4,35]$. An increase in cardiomyocyte size and protein synthesis is observed during physiological and pathological hypertrophy in response to either growth or stress signals, respectively [35]. These conditions differ in that only pathological hypertrophy is characterised by collagen accumulation and increased wall thickness, which compromises end-diastolic blood volume and is an independent predictor of cardiovascular death [36]. This is the first study using MRI to show that HIIT can stimulate positive cardiac remodelling.

The increase in stroke volume and ejection fraction is important because those with type 2 diabetes have reduced cardiac contractile capabilities [4]. Cardiomyocyte responses to high intensity exercise training in animal models have demonstrated improvements in the maximal extent of shortening, contraction and relaxation rates, with twice the improvement seen after training at $85-90 \%$ compared with training at $65-70 \% \dot{V} \mathrm{O}_{2 \text { peak }}$ [37]. The increased myofilament sensitivity 
Table 3 The effect of HIIT on body composition, blood variables and metabolic control

\begin{tabular}{|c|c|c|c|c|c|c|c|}
\hline \multirow[t]{2}{*}{ Parameter } & \multicolumn{3}{|l|}{ Control } & \multicolumn{3}{|l|}{ HIIT } & \multirow{2}{*}{$\begin{array}{l}\text { Adjusted between- } \\
\text { group } p \text { value }\end{array}$} \\
\hline & Pre & Post & $\begin{array}{l}\text { Within- } \\
\text { group } p \\
\text { value }^{\mathrm{a}}\end{array}$ & Pre & Post & $\begin{array}{l}\text { Within- } \\
\text { group } p \\
\text { value }^{\mathrm{a}}\end{array}$ & \\
\hline \multicolumn{8}{|l|}{ Body composition } \\
\hline Weight $(\mathrm{kg})$ & $90 \pm 9$ & $91 \pm 10$ & 0.06 & $90 \pm 15$ & $89 \pm 15$ & 0.09 & $0.02^{\dagger}$ \\
\hline Fat mass $(\mathrm{kg})$ & $35.6 \pm 10.9$ & $36.0 \pm 11.3$ & 0.36 & $31.9 \pm 9.3$ & $30.8 \pm 10.2$ & 0.09 & 0.08 \\
\hline Fat free mass $(\mathrm{kg})$ & $54.3 \pm 5.9$ & $54.7 \pm 5.7$ & 0.28 & $57.7 \pm 9.0$ & $58.2 \pm 8.9$ & 0.34 & 0.72 \\
\hline Visceral adipose tissue $\left(\mathrm{cm}^{2}\right)$ & $159 \pm 58$ & $156 \pm 49$ & 0.21 & $201 \pm 80$ & $181 \pm 72$ & $0.04 *$ & 0.08 \\
\hline Liver fat $(\%)$ & $7.1 \pm 6.8$ & $7.7 \pm 6.9$ & 0.12 & $6.9 \pm 6.9$ & $4.2 \pm 3.6$ & 0.06 & $0.01^{\dagger \dagger}$ \\
\hline \multicolumn{8}{|l|}{ Blood variables } \\
\hline $\operatorname{ALT}(\mathrm{U} / \mathrm{l})$ & $34 \pm 16$ & $33 \pm 14$ & 0.82 & $36 \pm 11$ & $30 \pm 10$ & $0.02 *$ & 0.14 \\
\hline $\operatorname{AST}(\mathrm{U} / \mathrm{l})$ & $27.6 \pm 10.4$ & $26.5 \pm 8.8$ & 0.63 & $27 \pm 7$ & $24 \pm 6$ & $0.02 *$ & 0.25 \\
\hline ALP (U/l) & $59.2 \pm 16.8$ & $61.2 \pm 17.5$ & 0.09 & $66 \pm 17$ & $63 \pm 16$ & 0.10 & $0.03^{\dagger}$ \\
\hline Total cholesterol $(\mathrm{mmol} / \mathrm{l})$ & $4.5 \pm 0.9$ & $4.6 \pm 0.9$ & 0.62 & $4.0 \pm 1.0$ & $4.5 \pm 1.1$ & 0.15 & 0.77 \\
\hline Triacylglycerol (mmol/l) & $1.1 \pm 0.4$ & $1.2 \pm 0.4$ & 0.12 & $1.1 \pm 0.3$ & $1.2 \pm 0.4$ & 0.28 & 0.87 \\
\hline \multicolumn{8}{|l|}{ Metabolic control } \\
\hline $\mathrm{HbA}_{1 \mathrm{c}}(\%)$ & $7.2 \pm 0.5$ & $7.4 \pm 0.7$ & 0.07 & $7.1 \pm 1.0$ & $6.8 \pm 0.9$ & 0.10 & $0.02^{\dagger}$ \\
\hline $\mathrm{HbA}_{1 \mathrm{c}}(\mathrm{mmol} / \mathrm{mol})$ & $54.9 \pm 5.9$ & $57.0 \pm 7.5$ & 0.06 & $54.5 \pm 10.6$ & $51.3 \pm 10.2$ & 0.09 & \\
\hline Fasting glucose $(\mathrm{mmol} / \mathrm{l})$ & $7.0 \pm 1.0$ & $7.6 \pm 1.4$ & $0.03 *$ & $6.8 \pm 1.6$ & $6.8 \pm 1.6$ & 0.87 & 0.15 \\
\hline Fasting insulin (pmol/1) & $81.5 \pm 46.4$ & $88 \pm 39.5$ & 0.42 & $65.5 \pm 39.5$ & $65.5 \pm 32.8$ & 0.88 & 0.22 \\
\hline $2 \mathrm{~h}$ glucose $(\mathrm{mmol} / \mathrm{l})$ & $11.7 \pm 3.1$ & $12.9 \pm 2.7$ & $0.01 * *$ & $12.5 \pm 3.1$ & $11.7 \pm 3.1$ & 0.22 & $0.02^{\dagger}$ \\
\hline $2 \mathrm{~h}$ AUGC & $1,366 \pm 66$ & $1,544 \pm 86$ & $0.01 * *$ & $1,395 \pm 81$ & $1,399 \pm 87$ & 0.94 & $0.02^{\dagger}$ \\
\hline HOMA-IR & $1.6 \pm 0.9$ & $1.8 \pm 0.8$ & 0.40 & $1.3 \pm 0.8$ & $1.4 \pm 0.6$ & 0.94 & 0.19 \\
\hline HOMA2- $\beta$ & $67.8 \pm 31.4$ & $67.0 \pm 37.3$ & 0.79 & $68.9 \pm 48.6$ & $70.9 \pm 49.0$ & 1.00 & 0.76 \\
\hline HOMA2-S & $76.1 \pm 33.0$ & $67.3 \pm 29.5$ & 0.25 & $101.7 \pm 48.1$ & $98.2 \pm 53.8$ & 0.88 & 0.39 \\
\hline
\end{tabular}

Data are mean \pm SD

${ }^{\text {a }}$ Paired $t$ test

${ }^{\mathrm{b}}$ Adjusted for baseline value for ANCOVA

* Significant difference baseline vs post-treatment $(p<0.05)$

$* *$ Significant difference baseline vs post-treatment $(p<0.01)$

${ }^{\dagger}$ Significant difference between-group interaction $(p<0.05)$

${ }^{\dagger}$ Significant difference between-group interaction $(p<0.01)$

Pre, pre-treatment; post, post-treatment

to calcium and the faster rise and diastolic decay of the calcium transient underpins these changes [37].

The early filling rate increased by $24 \%$, suggesting that the myocardium is more compliant and quicker to relax following HIIT. Diastolic dysfunction is the most widely reported malfunction of the diabetic heart and an independent predictor of mortality $[5,7,38]$, thus emphasising the clinical relevance of these findings. One study using echocardiography demonstrated diastolic improvements following 12 weeks of HIIT [14] and a longer term intervention using moderate exercise demonstrated improvements only when exercise was performed in the vigorous zone [39].

The decrease in torsion after HIIT provides, for the first time, evidence that exercise can be used to reverse the raised cardiac torsion observed in type 2 diabetes [8]. Cardiac torsion is a normal feature of contraction in a healthy heart and reflects the dominance of epicardial fibres over endocardial fibres [40]. Raised torsion in type 2 diabetes is a consequence of impaired contraction of endocardial fibres, which are less able to counteract this twisting motion [40]. These results indicate that endocardial damage and potential perfusion deficits at the endocardium can be improved with HIIT. No change was observed in peak endocardial and peak whole wall circumferential strain because the relative contribution of fibres across the myocardial wall remained constant, as reflected by the maintained eccentricity ratio.

HIIT stimulated improvements in cardiac structure and function independent of changes in cardiac metabolism. It 
was previously suggested that defects in cardiac metabolism underlie cardiac abnormalities seen in type 2 diabetes [7]; however, the decrease in PCr/ATP ratio in type 2 diabetes most likely reflects changes in substrate supply to the heart rather than an underlying metabolic defect in the myocardium [6].

Metabolic changes These data reveal for the first time that HIIT can reduce liver and visceral fat in type 2 diabetes, which is clinically important because both fat depots play a key pathogenic role in this chronic disease $[16,17]$. To our knowledge, this is the greatest reduction in liver fat to be reported following exercise in type 2 diabetes.

Despite this, fasting blood glucose did not change and, in line with data in healthy adults and obese women $[41,42]$, the results demonstrated no impact of HIIT on central insulin sensitivity in adults with type 2 diabetes. These results differ from those obtained after the very low energy diet $(2,512 \mathrm{~kJ}$ [600 kcal]), which led to a $30 \%$ relative reduction in liver fat and normalisation of fasting blood glucose after just 7 days [43]. In the present study, however, there was large interindividual variation in liver fat changes after HIIT, which may explain the absence of change in fasting blood glucose within this small sample. Indeed, those who lost the greatest liver fat had the largest reductions in fasting glucose, as reflected in the significant correlation.

It has been reported that HIIT acutely improves peripheral insulin sensitivity when measured within $72 \mathrm{~h}$ of the last exercise bout, attributable to rapid glycogen breakdown and subsequent re-synthesis [41]. The two studies which have measured postprandial response to HIIT in type 2 diabetes used continuous blood glucose measurements under standard dietary conditions [44, 45]. Dietary intake was not standardised in the present study, which may explain the lack of within-group improvement in $2 \mathrm{~h}$ glucose or HOMA-IR.

Within-group analysis also revealed no significant impact of HIIT on $\mathrm{HbA}_{1 \mathrm{c}}$. The significant between-group interactions most likely reflect a worsening of glycaemic control in the control group. $\mathrm{HbA}_{1 \mathrm{c}}$ was reduced by around $0.3 \%$ following HIIT, which is less than the reported mean effect $(0.6 \%$ reduction) of exercise interventions [33]. That being said, the greatest improvements in $2 \mathrm{~h}$ glucose and $\mathrm{HbA}_{1 \mathrm{c}}$ occurred in those who lost the largest amount of whole body fat mass and liver fat. As for fasting blood glucose, we speculate that $2 \mathrm{~h}$ glucose and $\mathrm{HbA}_{1 \mathrm{c}}$ within-group changes failed to reach significance because of the variation in liver fat reductions following HIIT.

This study questions the impact of HIIT on glycaemic control in adults with type 2 diabetes, but also confirms the importance of ectopic fat. Patients were required to maintain their weight during the HIIT programme, which may have compromised any improvements in glucose control. Interventions to target weight loss and ectopic fat may be most beneficial for glycaemic improvements. Despite this, these data highlight the positive impact of exercise on cardiac health, which may be improved further when accompanied by weight loss.

This study is not without limitation. The physiological mechanisms underlying the cardiac adaptations could not be elucidated with the MRI techniques used. Although magnetic resonance methods for quantifying ATP flux in the human heart by cardiac spectroscopy have been demonstrated in heart failure [46], they remain timeconsuming and prone to variability. The $\mathrm{PCr} / \mathrm{ATP}$ ratio can be measured in a reasonable period of time and has been demonstrated to be impaired in type 2 diabetes [47]. Using the RPE as a guide for exercise intensity (rather than an objective measure) may have limited the accuracy of the training intensity; however the RPE has been found to be an accurate predictor of exercise intensity in diabetes [48]. Finally, dietary monitoring was not adopted throughout the intervention.

In summary, this study demonstrates for the first time improvements in cardiac structure and function in patients with type 2 diabetes following a HIIT programme. These changes were accompanied by modest improvements in glycaemic control. HIIT elicited the greatest reduction in liver fat to be recorded following an exercise intervention in type 2 diabetes, demonstrating that this type of exercise is effective at targeting fat depots which play a role in the aetiology of this chronic condition. Although the direct benefits of HIIT to glycaemic control remain uncertain, HIIT is a potential therapy to moderate cardiac risk and reduce liver fat in type 2 diabetes and should be considered by clinicians alongside other regimens to improve glycaemic control.

Acknowledgements We thank the participants for contributing to this study. We acknowledge significant contribution from T. Hodgson, L. Morris and D. Wallace, research radiographers at the Magnetic Resonance Centre, Institute of Cellular Medicine, Newcastle upon Tyne, UK. Some of the data were presented as an abstract at the Diabetes UK Professional Conference, London, UK, 11-13 March 2015.

Funding KGH is supported by a Medical Research Council New Investigator Research Grant (G1100160), JP by the Magnetic Resonance Centre, Newcastle University, DGJ by the UKRC Centre for Ageing and Vitality, Newcastle University, and MIT by a National Institute for Health Research Senior Research Fellowship.

Duality of interest statement The authors declare that there is no duality of interest associated with this manuscript.

Contribution statement MIT, RT and KGH designed the study; $\mathrm{SC}, \mathrm{CT}$ and $\mathrm{KH}$ were responsible for data collection and acquisition; JP, KGH, RT, CT, DGJ and MIT assisted in data analysis and interpretation; and $\mathrm{SC}, \mathrm{KGH}$ and MIT wrote the manuscript. All authors critically reviewed the manuscript and approved the final version for publication. DGJ and MIT supervised the project. MIT is the guarantor of this work. 
Open Access This article is distributed under the terms of the Creative Commons Attribution 4.0 International License (http:// creativecommons.org/licenses/by/4.0/), which permits unrestricted use, distribution, and reproduction in any medium, provided you give appropriate credit to the original author(s) and the source, provide a link to the Creative Commons license, and indicate if changes were made.

\section{References}

1. IDF (2013) International Diabetes Federation Diabetes Atlas, 6th edn. IDF, Brussels

2. Reis SE, Holubkov R, Edmundowicz D et al (1997) Treatment of patients admitted to the hospital with congestive heart failure: specialty-related disparities in practice patterns and outcomes. J Am Coll Cardiol 30:733-738

3. Health and Social Care Information Centre (2013) National Diabetes Audit 2012-2013. Report 2: Complications and Mortality. HSCIC, Leeds, pp 1-25

4. Dawson A, Morris AD, Struthers AD (2005) The epidemiology of left ventricular hypertrophy in type 2 diabetes mellitus. Diabetologia 48:1971-1979

5. Rijzewijk LJ, van der Meer RW, Lamb HJ et al (2009) Altered myocardial substrate metabolism and decreased diastolic function in nonischemic human diabetic cardiomyopathy: studies with cardiac positron emission tomography and magnetic resonance imaging. J Am Coll Cardiol 54:1524-1532

6. Cassidy S, Hallsworth K, Thoma C et al (2015) Cardiac structure and function are altered in type 2 diabetes and nonalcoholic fatty liver disease and associate with glycemic control. Cardiovasc Diabetol 14:23

7. Diamant M, Lamb HJ, Groeneveld Y et al (2003) Diastolic dysfunction is associated with altered myocardial metabolism in asymptomatic normotensive patients with well-controlled type 2 diabetes mellitus. J Am Coll Cardiol 42:328-335

8. Fonseca CG, Dissanayake AM, Doughty RN et al (2004) Threedimensional assessment of left ventricular systolic strain in patients with type 2 diabetes mellitus, diastolic dysfunction, and normal ejection fraction. Am J Cardiol 94:1391-1395

9. Grothues F, Smith GC, Moon JCC et al (2002) Comparison of interstudy reproducibility of cardiovascular magnetic resonance with two-dimensional echocardiography in normal subjects and in patients with heart failure or left ventricular hypertrophy. Am J Cardiol 90:29-34

10. Inzucchi SE, Bergenstal RM, Buse JB et al (2012) Management of hyperglycemia in type 2 diabetes: a patient-centered approach: position statement of the American Diabetes Association (ADA) and the European Association for the Study of Diabetes (EASD). Diabetes Care 35:1364-1379

11. Chudyk A, Petrella RJ (2011) Effects of exercise on cardiovascular risk factors in type 2 diabetes: a meta-analysis. Diabetes Care 34: $1228-1237$

12. Gibala MJ, Little JP, Macdonald MJ, Hawley JA (2012) Physiological adaptations to low-volume, high-intensity interval training in health and disease. J Physiol 590:1077-1084

13. Wisløff U, Støylen A, Loennechen JP et al (2007) Superior cardiovascular effect of aerobic interval training versus moderate continuous training in heart failure patients: a randomized study. Circulation 115:3086-3094

14. Hollekim-Strand SM, Bjørgaas MR, Albrektsen G et al (2014) High-intensity interval exercise effectively improves cardiac function in patients with type 2 diabetes mellitus and diastolic dysfunction: a randomized controlled trial. J Am Coll Cardiol 64:17581760
15. Mitranun W, Deerochanawong C, Tanaka H, Suksom D (2014) Continuous vs interval training on glycemic control and macro-and microvascular reactivity in type 2 diabetic patients. Scand J Med Sci Sports 24:e69-e76

16. Björntorp P (1991) Metabolic implications of body fat distribution. Diabetes Care 14:1132-1143

17. Taylor R (2013) Type 2 diabetes: etiology and reversibility. Diabetes Care 36:1047-1055

18. ACSM, Thompson WR, Gordon NF, Pescatello LS (2010) ACSM's guidelines for exercise testing and prescription, 8th edn. Wolters Kluwer/Lippincott Williams \& Wilkins, Philadelphia

19. Gratze G, Fortin J, Holler A et al (1998) A software package for non-invasive, real-time beat-to-beat monitoring of stroke volume, blood pressure, total peripheral resistance and for assessment of autonomic function. Comput Biol Med 28:121-142

20. Jones DEJ, Hollingsworth K, Fattakhova G et al (2010) Impaired cardiovascular function in primary biliary cirrhosis. Am J Physiol Gastrointest Liver Physiol 298:G764-G773

21. Hollingsworth KG, Blamire AM, Keavney BD, Macgowan GA (2012) Left ventricular torsion, energetics, and diastolic function in normal human aging. Am J Physiol Heart Circ Physiol 302: $\mathrm{H} 885-\mathrm{H} 892$

22. Schär M, Vonken E-J, Stuber M (2010) Simultaneous B(0)-and $\mathrm{B}(1)+$-map acquisition for fast localized shim, frequency, and RF power determination in the heart at 3T. Magn Reson Med 63:419426

23. Vanhamme L, Van Huffel S, Van Hecke P, van Ormondt D (1999) Time-domain quantification of series of biomedical magnetic resonance spectroscopy signals. J Magn Reson 140:120-130

24. Bottomley PA (2009) NMR spectroscopy of the human heart. eMagRes. doi:10.1002/9780470034590.emrstm0345.pub2

25. Szczepaniak LS, Nurenberg P, Leonard D et al (2005) Magnetic resonance spectroscopy to measure hepatic triglyceride content: prevalence of hepatic steatosis in the general population. Am J Physiol Endocrinol Metab 288:E462-E468

26. Shen W, Punyanitya M, Wang Z et al (2004) Visceral adipose tissue: relations between single-slice areas and total volume. Am J Clin Nutr 80:271-278

27. Donnelly LF, O’Brien KJ, Dardzinski BJ et al (2003) Using a phantom to compare MR techniques for determining the ratio of intraabdominal to subcutaneous adipose tissue. AJR Am J Roentgenol 180:993-998

28. Abramoff MD, Magalhães PJ, Ram SJ (2004) Image processing with ImageJ. Biophoton Int 11:36-42

29. Levy JC, Matthews DR, Hermans MP (1998) Correct homeostasis model assessment (HOMA) evaluation uses the computer program. Diabetes Care 21:2191-2192

30. Floch J-P, Escuyer P, Baudin E et al (1990) Blood glucose area under the curve. Methodological aspects. Diabetes Care 13:172175

31. Borg GAV (1982) Psychophysical bases of perceived exertion. Med Sci Sports Exerc 14:377-381

32. St-Onge M, Mignault D, Allison DB, Rabasa-Lhoret R (2007) Evaluation of a portable device to measure daily energy expenditure. Am J Clin Nutr 742-749

33. Thomas DE, Elliott EJ, Naughton GA (2006) Exercise for type 2 diabetes mellitus. Cochrane Database Syst Rev, Issue 3, Art. no.: CD002968. doi:10.1002/14651858.CD002968.pub2

34. Rothman KJ (1990) No adjustments are needed for multiple comparisons. Epidemiology 1:43-46

35. Frey N, Katus HA, Olson EN, Hill JA (2004) Hypertrophy of the heart: a new therapeutic target? Circulation 109:1580-1589

36. Liao Y, Cooper RS, McGee DL et al (1995) The relative effects of left ventricular hypertrophy, coronary artery disease, and ventricular dysfunction on survival among black adults. JAMA 273:15921597 
37. Kemi OJ, Haram PM, Loennechen JP et al (2005) Moderate vs. high exercise intensity: differential effects on aerobic fitness, cardiomyocyte contractility, and endothelial function. Cardiovasc Res 67:161-172

38. Halley CM, Houghtaling PL, Khalil MK et al (2011) Mortality rate in patients with diastolic dysfunction and normal systolic function. Arch Intern Med 171:1082-1087

39. Hordern MD, Coombes JS, Cooney LM et al (2009) Effects of exercise intervention on myocardial function in type 2 diabetes. Heart 95:1343-1349

40. Lumens J, Delhaas T, Arts T et al (2006) Impaired subendocardial contractile myofiber function in asymptomatic aged humans, as detected using MRI. Am J Physiol Heart Circ Physiol 291: H1573-H1579

41. Babraj JA, Vollaard NBJ, Keast C et al (2009) Extremely short duration high intensity interval training substantially improves insulin action in young healthy males. BMC Endocr Disord 9:3

42. Gillen JB, Percival ME, Ludzki A et al (2013) Interval training in the fed or fasted state improves body composition and muscle oxidative capacity in overweight women. Obesity (Silver Spring) 21: 2249-2255
43. Lim EL, Hollingsworth KG, Aribisala BS et al (2011) Reversal of type 2 diabetes: normalisation of beta cell function in association with decreased pancreas and liver triacylglycerol. Diabetologia 54: 2506-2514

44. Little JP, Gillen JB, Percival ME et al (2011) Low-volume highintensity interval training reduces hyperglycemia and increases muscle mitochondrial capacity in patients with type 2 diabetes. J Appl Physiol 111:1554-1560

45. Gillen JB, Little JP, Punthakee Z et al (2012) Acute high-intensity interval exercise reduces the postprandial glucose response and prevalence of hyperglycaemia in patients with type 2 diabetes. Diabetes Obes Metab 14:575-577

46. Weiss RG, Gerstenblith G, Bottomley PA (2005) ATP flux through creatine kinase in the normal, stressed, and failing human heart. Proc Natl Acad Sci U S A 102:808-813

47. Scheuermann-Freestone M, Madsen PL, Manners D et al (2003) Abnormal cardiac and skeletal muscle energy metabolism in patients with type 2 diabetes. Circulation 107:3040 3046

48. Colberg SR, Swain DP, Vinik AI (2003) Use of heart rate reserve and rating of perceived exertion to prescribe exercise intensity in diabetic autonomic. Diabetes Care 26:986-990 